\title{
Coping e saúde mental de adolescentes vestibulandos
}

\author{
Lorena Soares Dias e Silva \\ Daniela S. Zanini \\ Pontificia Universidade Católica de Goiás
}

\begin{abstract}
Resumo
Este estudo teve como objetivo investigar o uso de estratégias de enfrentamento de problemas (coping) por adolescentes vestibulandos e sua possível repercussão na saúde mental desses indivíduos. Participaram deste estudo 292 estudantes, sendo 59,6\% mulheres e 38,0\% homens com idades entre 16 e 19 anos $(M=$ $17,09 ; D P=0,71)$, contatados em escolas públicas, particulares e conveniadas, que declararam intenção de realizar o processo seletivo vestibular. Foram utilizados o Coping Response Inventory Youth Form (CRI-Y) para avaliar as estratégias de coping e o Youth Self Report (YSR) para avaliar as psicopatologias. O estudo demonstrou que os adolescentes avaliaram o vestibular como evento estressante, e aqueles que utilizaram mais estratégias de coping de aproximação para enfrentá-lo relataram menos psicopatologias. Os resultados deste estudo põem em evidência a relação entre vestibular como evento estressante e saúde mental dos adolescentes.
\end{abstract}

Palavras-chave: coping; psicopatologia; vestibulandos.

\begin{abstract}
Coping and mental health in adolescents preparing for the Brazilian university entrance examination. This study aimed to investigate the use of coping strategies by adolescents preparing to take the Brazilian university entrance exam and its possible repercussion on their mental health. A total of 292 students participated in the study, 59.6\% women and 38.0\% men, aged between 16 and 19 years $(M=17.09 ; S D=0.71)$, contacted in public, private, and private schools with state government covenant, who declared the intention to take the Brazilian university entrance exam. The Coping Response Inventory Youth Form (CRI-Y) was used to assess coping strategies and the Youth Self Report (YSR) to evaluate psychopathologies. The study demonstrated that the adolescents' appraise the Brazilian university entrance exam as a stressful event, and those who used more approach coping strategies reported less psychopathologies. The results of this study highlighted the relationship between the Brazilian university entrance exam as a stressful event and the adolescents' mental health.
\end{abstract}

Keywords: coping; psychopathology; university entrance exam.

$\mathrm{N}$ esta pesquisa, considera-se a adolescência como um momento de fragilidade para alguns indivíduos, potencializando o surgimento de eventuais crises decorrentes de mudanças físicas, psicológicas, sociais e culturais (Wagner, Falcke, Silveira, \& Mosmann, 2002). Dessa maneira, o vestibular pode ser entendido nesse processo como um dos elementos geradores ou agravadores de crise, podendo ser um evento estressante para o adolescente (Calais, Andrade, \& Lipp, 2003). Pesce, Assis, Santos e Oliveira (2004) conceituam eventos estressantes como riscos ou obstáculos individuais e ambientais que aumentam a vulnerabilidade do indivíduo ao desencadeamento de problemas de saúde.

Silvestre (2004) e Sparta e Gomes (2005) discutem em seus estudos a importância atribuída ao vestibular por parte dos alunos em decorrência de seu desejo de obter ascensão social propiciada pela formação acadêmica, o que torna este tipo de exame um evento ansiogênico por seu papel classificatório e excludente.
Assim, o nível superior é entendido pelos jovens de baixa renda como uma oportunidade de ascensão social. Contudo, para os jovens de classe média, a faculdade é uma condição sine qua non para garantir a manutenção do nível socioeconômico. Portanto, o que poderia ou deveria ser um instrumento de inclusão, acaba tornando-se instrumento de exclusão social, não por seu papel discriminativo em si, mas porque reflete as desigualdades educacionais consequentes às desigualdades econômicas. Desde a sua criação, na década de 1950, passando por ajustes e adequações nos anos 60, a verdadeira função do vestibular deveria ser de "[...] avaliar a formação recebida pelos candidatos e sua aptidão intelectual para estudos superiores [...]", conforme o Art. 21 da Lei $n^{\circ}$ 5.540/68 (1968). Percebe-se um paradoxo entre o que está na Lei de Diretrizes e Bases da Educação (Lei $\left.\mathrm{n}^{\mathrm{o}} 9.394,1996\right)$ - a teoria - e o que ocorre durante o processo seletivo - a prática. Isso porque, enquanto a lei determina que o ensino médio propicie educação totalizada, de modo que 
o indivíduo possa exercer sua cidadania de forma crítica e consciente, na prática, o ensino focaliza seu objetivo cada vez mais na preparação da pessoa exclusivamente para participar do processo seletivo do vestibular.

Quando o jovem não dispõe de mecanismos internos e/ ou externos para lidar com a situação estressante propiciada pelo vestibular, pode haver o desencadeamento de uma série de psicopatologias, como depressão e ansiedade (Zanini, Forns, \& Kirchner, 2005) e distúrbios do humor (Peluso, Savalli, Cúri, Gorestein, \& Andrade, 2010). Nesse último caso, Peluso et al. (2010) demonstraram que embora as alterações do humor sofridas pelos adolescentes ocorram previamente ao enfrentamento do vestibular, tendem a persistir meses após a vivência deste evento.

Nessa perspectiva, percebe-se que não apenas a vivência dos eventos estressantes, mas também a maneira como os adolescentes os enfrentam podem contribuir para eventual aumento ou diminuição de suas consequências. Em vista disso, Calais et al. (2003) postularam que a habilidade em lidar com as situações de estresse e ansiedade por parte dos adolescentes podem ser fatores diferenciais e mais importantes do que a habilidade acadêmica ou o conhecimento em relação ao enfrentamento da situação de vestibular. Essas estratégias de enfrentamento também são chamadas de coping, que pode ser conceituado como os esforços cognitivos e comportamentais utilizados pelos indivíduos a fim de lidar com os eventos avaliados como excedentes à sua capacidade de fazer frente a eles (Lazarus \& Folkman, 1984; Moos, 1993).

O conceito de coping é derivado de duas abordagens: a primeira, vinculada à experimentação animal, refere-se ao comportamento de fuga e esquiva; e a segunda, relacionada à psicologia do ego, está focada nos mecanismos de defesa propostos pela psicanálise (Antoniazzi, Dell'Aglio, \& Bandeira, 1998; Savoia, 1999). Em uma visão mais integradora, o coping é explicado como um processo transacional entre a pessoa e o ambiente, em que as estratégias de enfrentamento têm as funções de alterar a relação entre a pessoa e o ambiente e adequar a resposta emocional ao problema (Zanini \& Forns, 2004).

Ainda sob essa óptica, o coping pode ser também entendido em um modelo transacional classificando-se as respostas ou estratégias empregadas pelo indivíduo em dois grupos, de acordo com o foco: estratégias de enfrentamento focalizadas no problema e estratégias de enfrentamento focalizadas na emoção. Essas nomenclaturas podem variar com o pesquisador, citando-se como exemplo Moos (1993), que embora mantenha os mesmos conceitos, define essas estratégias atribuindo-lhes outras denominações: para coping com foco no problema, usa o termo coping de aproximação e para coping com foco na emoção, utiliza o termo coping evitativo. O primeiro seria a tentativa de modificar, cognitiva ou comportamentalmente, a situação estressora e o segundo tem o objetivo de reduzir a sensação física desagradável desse estado de estresse (Faria \& Seidl, 2005).

Dell'Aglio (2003) discutiu a influência que as formas de enfrentamento de problemas podem ter sobre a saúde mental dos jovens. Por seu turno, Câmara e Carlotto (2007) demonstraram associação entre uso de estratégias de coping do tipo resolução de problemas pelas meninas e maior índice de bem estar psicológico. Além disso, estudos mostram a existência de diferentes respostas de coping e percepção do problema em relação a sexo, faixa etária, nível socioeconômico, entre outros fatores. Pode-se citar como exemplos que: as meninas utilizam estratégias focalizadas na emoção com maior frequência do que os meninos; e os adolescentes mais jovens (entre 11 e 13 anos) tendem a usar mais estratégias comportamentais, enquanto os adolescentes mais velhos (entre 14 e 18 anos) tendem a usar mais estratégias cognitivas (Dell'Aglio \& Hutz, 2002; Moos, 1993; Zanini \& Forns, 2004). Por conseguinte, deve-se levar em conta a variabilidade biopsicossociocultural do indivíduo para compreender a forma como ele interage com o meio.

Para estudar coping em adolescentes, é preciso fazer uma adaptação ao contexto de vida do adolescente e às teorias do estresse aplicadas a este contexto vivencial. Assim, não basta apenas importar a teoria de estresse e coping existente para a população adulta e aplicá-la ao contexto do adolescente. As diferenças dos recursos de coping, de relacionamentos sociais, entre outros, justificam a necessidade de desenvolvimento de teorizações próprias para esta etapa evolutiva (Zanini et al., 2005). Para isso, faz-se necessário compreender o vínculo dos adolescentes com os adultos, a sua interação com o ambiente, as suas habilidades cognitivas e comportamentais, entre outros recursos situacionais e disposicionais (Compas, 1987). Como mencionado anteriormente, a adolescência é um período vulnerável por conta de múltiplos acontecimentos internos e externos ao jovem (Reitz, Dekovic, \& Meijer 2005), potencializando o surgimento de uma variedade de psicopatologias.

Stevens, Vollebergh, Pels e Crijnen (2005), em sua pesquisa na Holanda com adolescentes imigrantes do Marrocos, constataram que o fator cultural teve forte predição na aparição de sintomas internalizantes (comportamentos relativos a afastamento, queixas somáticas, ansiedade e depressão) e externalizantes (comportamentos delinquentes e condutas agressivas) (Achenbach, 1991). Observaram maior predição de fatores internalizantes em meninas e atribuíram este resultado à diferença de liberdade entre meninas e meninos, acrescentando que o afeto e a proteção familiar podem ser fatores de proteção contra problemas emocionais. No presente estudo, o fator cultural foi trabalhado de modo genérico, referindo-se à importância que a cultura brasileira, em geral, tem dado ao vestibular como instrumento de seleção de indivíduos que cursarão o ensino superior.

Assim, neste estudo, pretendeu-se investigar o uso de estratégias de coping por adolescentes vestibulandos e suas consequências para a saúde mental destes jovens. Mais especificamente, no presente estudo pretendeu-se avaliar o uso de estratégias pelos adolescentes para o enfrentamento da situação estressante vestibular segundo o sexo, a idade e o tipo de escola que frequentam e sua influência na saúde mental destes indivíduos.

Estudos anteriores apontaram para a relevância do estudo das habilidades de coping como possível fator determinante para o sucesso no exame vestibular quando comparado com o desempenho acadêmico (Calais et al., 2003). Diante disso, o estudo da relação entre uso de coping e manifestações 
psicopatológicas poderia lançar luz nas estratégias de intervenções psicológicas mais efetivas para o tratamento de adolescentes que se submetem ao vestibular e, desta forma, apresentam risco para manifestações psicopatológicas ou mal-estar psicológico (Peluso et al., 2010), problemas de sono (Rocha, Rossini, \& Reimão, 2010), entre outros.

\section{Método}

\section{Participantes}

Participaram deste estudo 292 adolescentes contatados em instituições de ensino médio de Goiânia-GO, selecionados por conglomerados, a fim de representar a população adolescente da cidade. Desses, $11,3 \%$ estavam frequentando o terceiro ano de um colégio estadual localizado em um bairro de classe popular, $46,2 \%$ eram alunos do terceiro ano do ensino médio de um colégio privado em um bairro de classe média alta, $21,2 \%$ cursavam o terceiro ano do ensino médio de uma escola da rede particular de ensino conveniada (cuja mensalidade dos alunos era parcialmente subsidiada por verbas públicas) em um bairro de classe média baixa e $20,5 \%$ eram alunos do curso preparatório para vestibular de uma escola da rede particular de ensino em um bairro de classe média, enquanto $0,8 \%$ dos participantes omitiram esta informação.

Para este estudo, apenas foram acessados os adolescentes que informaram interesse em se preparar para realizar o exame vestibular, sendo $174(59,6 \%)$ do sexo feminino, $111(38,0 \%)$ do sexo masculino e $7(2,4 \%)$ não informaram o sexo, com idades compreendidas entre 16 e 19 anos $(M=17,09 ; D P=0,71)$. Foram excluídos aqueles protocolos cujos indivíduos relataram: ocorrência de gestação, uso de drogas ou não ter a intenção de prestar vestibular. Os casos relativos à gestação ou uso de drogas foram encaminhados ao serviço de psicologia da universidade a que se vincula esta pesquisa, conforme estabelecido no Projeto aprovado pela Comissão Nacional de Ética-CONEP (protocolo $\mathrm{n}^{\mathrm{o}}$ 0113.0.168.000-06).

\section{Instrumentos}

As estratégias de coping foram avaliadas utilizando-se o Coping Response Inventory Youth Form (CRI-Y), de Moos (1993). Este inventário está em fase de validação para a população brasileira; porém, estudos preliminares apontam que os coeficientes alfa das escalas da adaptação brasileira são similares aos da versão inglesa, variando de 0,45 a 0,67 para meninos e de 0,50 a 0,72 para meninas, para as escalas diretas, e 0,79 e 0,76 para meninos e 0,79 e 0,71 para meninas nas escalas globais (Zanini, Mendonça, Forns, \& Kirchner, 2010). Embora os coeficientes alfa das escalas diretas desse instrumento possam ser classificados como fracos, estão em consonância com os descritos na literatura mundial do tema. Além disso, os índices satisfatórios encontrados para as escalas globais, a vasta utilização desse instrumento em diferentes culturas e a solidez da teoria que o fundamenta justificam a sua escolha.

O CRI-Y é dividido em três partes. A primeira consiste na descrição de uma situação difícil ou estressante vivenciada nos últimos 12 meses, que no caso deste estudo é o exame vestibular.
A segunda é composta por dez perguntas sobre a percepção do problema vivenciado pelo sujeito, as quais foram consideradas em diversas perspectivas avaliadas e analisadas pelos participantes da pesquisa. A terceira compõe-se de 48 questões objetivas, as quais caracterizam as formas de enfrentamento de problemas utilizadas pelos adolescentes.

A partir de uma análise fatorial das 48 perguntas referentes à terceira parte do instrumento, tem-se a composição das oito estratégias de enfrentamento de problemas avaliadas pelo questionário, que são: a) aproximação cognitiva - análise lógica e reavaliação positiva; b) aproximação comportamental - busca de guia (referente à busca de apoio de alguém, como pais, pares ou adultos significativos) e resolução de problemas; c) evitação cognitiva, - evitação cognitiva e aceitação e resignação; d) evitação comportamental - busca de recompensa alternativa e descarga emocional (Holahan, Moos, \& Schaefer, 1996).

Para avaliar os comportamentos desadaptados, foi utilizado o Youth Self Report (YSR), questionário desenvolvido para adolescentes entre 11 e 18 anos de idade por Achenbach (1991) para obter respostas de adolescentes a respeito de suas próprias competências e problemas. Esse instrumento é composto por duas partes. A primeira contém sete questões que analisam comportamentos sociais dos adolescentes, tais como atividades cotidianas, amizades, hábitos sociais e familiares e rendimento escolar, formando a escala de competência. A segunda é composta por 112 itens que avaliam problemas de comportamento e comportamentos socialmente desejáveis, formando as escalas de distúrbio total e síndromes, referindo-se a sintomas psicopatológicos de naturezas distintas baseados no DSM-IVTR (American Psychiatric Association, 2002). Essas escalas são posteriormente agrupadas em dois grandes grupos denominados síndromes internalizantes (manifestações sindrômicas de afastamento, queixas somáticas, ansiedade e depressão) e síndromes externalizantes (compostas por comportamentos delinquentes e condutas agressivas) (Achenbach, 1991). Os adolescentes podem ser avaliados nas escalas individuais, das grandes síndromes (internalizantes e externalizantes), e também em relação ao distúrbio total. O resultado é ponderado em função do sexo e da idade dos respondentes.

O YSR está empiricamente baseado em dados de pesquisas desenvolvidas em diversos contextos e culturas (Achenbach, 1991). No Brasil, a bateria Achenbach System of Empirically Based Assessment (ASEBA), na qual o instrumento do YSR encontra-se inserido, está sendo traduzida e validada por um grupo de pesquisa da Universidade de Sâo Paulo e os dados preliminares indicam boa adequação psicométrica do instrumento (Bordin, Mari, \& Carero, 1995; Silva, Aznar-Farias, Silvares, \& Arantes, 2008).

Para este estudo, apenas a segunda parte do instrumento, relativa a perguntas fechadas, foi analisada e, mais especificamente, analisaram-se as síndromes externalizantes, internalizantes e a pontuação total no questionário. A primeira parte, referente às habilidades sociais dos adolescentes, foi reservada para estudos posteriores com metodologia qualitativa, pois utiliza perguntas abertas, o que exige outro nível de análise que não a aplicada neste estudo. 


\section{Procedimento}

Mediante a autorização das escolas para a execução da pesquisa, foram programadas três sessões para a assinatura do termo de consentimento livre e esclarecido e a aplicação dos questionários. Os questionários foram administrados de forma coletiva durante o período de aula dos alunos, aplicados por estudantes e psicólogos previamente orientados e treinados para esta finalidade.

Na primeira sessão, os alunos foram esclarecidos acerca da pesquisa e do sigilo. Oportunamente, abriu-se espaço para que quaisquer dúvidas a respeito dos instrumentos, da leitura e da assinatura do termo de consentimento livre e esclarecido fossem dirimidas. Na segunda e na terceira sessões, foram aplicados os questionários CRI-Y e YSR, respectivamente, respeitando-se as normas e as instruções de aplicação contidas nos manuais de cada um deles. O tempo para respondê-los foi livre, tendo variado de 15 minutos a 1 hora.

Embora este estudo não tenha tido caráter intervencionista, foi oferecida a todos os participantes da pesquisa que demonstraram interesse a possibilidade de apoio psicológico, o que foi registrado no protocolo $\mathrm{n}^{\circ}$ 0113.0.168.000-06. Os pais de todos os participantes deste estudo deram sua autorização, mediante assinatura do termo de consentimento livre e esclarecido, para a participação dos adolescentes.

\section{Resultados}

Para a apresentação dos dados observados, optou-se por dividir os resultados em duas partes, de acordo com as análises e as comparações entre grupos. Na primeira, descrevem-se percepção do problema, coping e psicopatologia em relação às variáveis sexo, idade e tipo de escola. Na segunda, é descrita a relação entre o uso de coping e o desenvolvimento de psicopatologias (internalizantes, externalizantes e total).
Os dados provenientes da aplicação dos instrumentos foram analisados utilizando-se o pacote estatístico para Windows SPSS versão 12.0 .

\section{Percepção do problema, coping e psicopatologia por sexo, idade e tipo de escola}

Inicialmente, procedeu-se à comparação de médias por meio da técnica estatística ANOVA entre as dez primeiras perguntas do CRI-Y, referentes à percepção do problema, e o uso das estratégias de coping segundo as variáveis sexo, idade e tipo de escola. Por meio dessas análises, buscou-se avaliar a influência das variáveis sociodemográficas sobre a percepção do problema ou o uso de estratégias de coping. Em todas as tabelas apresentadas são mostrados apenas os dados que foram estatisticamente significativos $(p<0,05)$.

Conforme observado na Tabela 1 , as meninas relataram ter experienciado significativamente mais do que os meninos "alguma coisa boa ao enfrentar o problema" $(F=4,24 ; p=0,05)$, enquanto os meninos relataram significativamente mais uso de “já resolvido o problema” $(F=6,85 ; p=0,01)$.

Analisando-se as estratégias, verificou-se que as meninas utilizaram significativamente mais estratégias de coping, do tipo análise lógica $(F=6,36 ; p=0,01)$, reavaliação positiva $(F=7,17$; $p=0,01)$, busca de guia $(F=7,64 ; p=0,01)$ e descarga emocional $(F=11,41 ; p=0,00)$ como forma de resolver seus problemas.

Em relação à percepção do problema e à idade, observou-se que os adolescentes de 18 anos $(M=1,48 ; D P=1,28)$ relataram significativamente mais do que seus companheiros de 17 anos $(M=1,03 ; D P=1,19)$ que "está tudo bem após o enfrentamento do problema" $(F=2,84 ; p=0,04)$, ao passo que os de 16 anos $(M=2,03 ; D P=1,27)$ relataram mais do que os de 17 anos $(M$ $=1,89 ; D P=1,19), 18$ anos $(M=1,51 ; D P=1,33)$ e $19 \operatorname{anos}(M$ $=0,83 ; D P=0,98)$ que ocorreu "alguma coisa boa ao enfrentar o problema" $(F=3,40 ; p=0,02)$. A análise das estratégias

Tabela 1

Dados estatisticamente significativos referentes à comparação de médias (M) e desvio padrão (DP) das variáveis sexo, percepção do problema e estratégias de coping utilizando-se ANOVA

\begin{tabular}{|c|c|c|c|c|}
\hline \multirow[b]{2}{*}{ Variável } & \multicolumn{4}{|c|}{ Sexo } \\
\hline & $\begin{array}{c}\text { Masculino } \\
M(D P)\end{array}$ & $\begin{array}{c}\text { Feminino } \\
M(D P)\end{array}$ & $F$ & $p<$ \\
\hline \multicolumn{5}{|l|}{ Percepção do problema } \\
\hline Alguma coisa boa ao enfrentar o problema & $1,6(1,3)$ & $1,9(1,2)$ & 4,2 & $0,05^{*}$ \\
\hline Já resolvido o problema & $1,2(1,5)$ & $0,8(1,2)$ & 6,9 & $0,01 * *$ \\
\hline \multicolumn{5}{|l|}{ Estratégia de coping } \\
\hline Análise lógica & $10,6(3,2)$ & $11,7(3,0)$ & 6,3 & $0,01 * *$ \\
\hline Reavaliação positiva & $10,6(3,8)$ & $11,8(3,4)$ & 7,2 & $0,01 * *$ \\
\hline Busca de guia & $7,5(4,4)$ & $9,0(4,5)$ & 7,6 & $0,01 * *$ \\
\hline Descarga emocional & $6,9(3,8)$ & $8,5(3,7)$ & 11,4 & $0,00 * * *$ \\
\hline
\end{tabular}

de coping por idade não revelou diferenças estatisticamente significativas.

Em relação à percepção do problema de acordo com o tipo de escola (Tabela 2), observaram-se diferenças significativas para os seguintes quesitos: "já teve de resolver um problema como este?" $(F=7,96 ; p=0,00)$, "achava que este problema poderia acontecer com você?" $(F=4,16 ; p=0,01)$, "teve tempo suficiente para a preparação?” $(F=3,21 ; p=0,02)$, "pensou de que maneira poderia acontecer com você?" $(F=3,83 ; p=0,01)$, "foi causado por você?" $(F=3,10 ; p=0,03)$, “já foi resolvido?" $(F=2,78$; $p=0,04)$ e "está tudo bem com você?" $(F=4,53 ; p=0,00)$.

$\mathrm{Na}$ Tabela 2, apresentam-se os dados da comparação de média segundo o tipo de escola. Observou-se diferença significativa entre o tipo de escola e as seguintes estratégias de 
Tabela 2

Dados estatisticamente significativos referentes à comparação de médias (M) e desvio padrão (DP) das variáveis tipo de escola, percepção do problema e estratégias de coping utilizando-se ANOVA

\begin{tabular}{|c|c|c|c|c|c|c|}
\hline \multirow[b]{2}{*}{ Variável } & \multicolumn{4}{|c|}{ Tipo de escola } & \multirow[b]{2}{*}{$F$} & \multirow[b]{2}{*}{$p<$} \\
\hline & $\begin{array}{c}\text { Pública } \\
3^{\circ} \text { ano } \\
M(D P)\end{array}$ & $\begin{array}{c}\begin{array}{c}\text { Particular } \\
3^{\circ} \text { ano }\end{array} \\
M(D P) \\
\end{array}$ & $\begin{array}{c}\text { Conveniada } \\
3^{\circ} \text { ano }\end{array}$ & $\begin{array}{c}\begin{array}{c}\text { Particular } \\
\text { cursinho }\end{array} \\
M(D P) \\
\end{array}$ & & \\
\hline \multicolumn{7}{|l|}{ Percepção do problema } \\
\hline $\begin{array}{l}\text { Já teve de resolver um problema } \\
\text { como este? }\end{array}$ & $1,3(1,3)^{b}$ & $0,4(0,8)^{\mathrm{a}}$ & $0,9(1,2)^{b}$ & $0,8(1,2)$ & 8,0 & $0,00 * * *$ \\
\hline $\begin{array}{l}\text { Achava que este problema } \\
\text { poderia acontecer com você? }\end{array}$ & $2,6(0,9)$ & $2,8(0,7)^{\mathrm{a}}$ & $2,3(1,2)^{b}$ & $2,4(1,0)$ & 4,2 & $0,01 * *$ \\
\hline $\begin{array}{l}\text { Teve tempo suficiente para a } \\
\text { preparação? }\end{array}$ & $0,8(1,2)^{b}$ & $1,5(1,3)$ & $1,3(1,3)$ & $1,6(1,2)^{\mathrm{a}}$ & 3,2 & $0,02 *$ \\
\hline $\begin{array}{l}\text { Pensou de que maneira poderia } \\
\text { prejudicar você? }\end{array}$ & $2,4(1,1)^{b}$ & $1,7(1,3)^{\mathrm{a}}$ & $2,0(1,3)$ & $2,1(1,0)$ & 3,8 & $0,01 * *$ \\
\hline Foi causado por você? & $0,8(1,1)$ & $0,9(1,1)$ & $1,2(1,3)$ & $1,4(1,3)$ & 3,1 & $0,03 *$ \\
\hline Já foi resolvido? & $1,4(1,5)$ & $0,8(1,3)$ & $1,2(1,3)$ & $1,1(1,1)$ & 2,8 & $0,04 *$ \\
\hline Está tudo bem com você? & $1,7(1,4)^{\mathrm{b}}$ & $0,9(1,2)^{\mathrm{a}}$ & $1,3(1,2)$ & $1,4(1,2)$ & 4,5 & $0,00 * * *$ \\
\hline \multicolumn{7}{|l|}{ Estratégia de coping } \\
\hline Análise lógica & $10,3(3,4)$ & $11,9(2,9)$ & $10,7(3,2)$ & $11,4(3,2)$ & 3,7 & $0,01 * *$ \\
\hline $\begin{array}{l}\text { Busca de guia } \\
\text { Descarga emocional }\end{array}$ & $\begin{array}{l}5,9(3,9)^{\mathrm{b}} \\
5,4(3,0)^{\mathrm{b}}\end{array}$ & $\begin{array}{l}9,6(4,5)^{\mathrm{a}} \\
8,8(3,7)^{\mathrm{a}}\end{array}$ & $\begin{array}{l}7,3(4,7)^{\mathrm{b}} \\
7,2(3,8)\end{array}$ & $\begin{array}{l}8,0(4,0) \\
7,8(3,6)^{\mathrm{a}}\end{array}$ & $\begin{array}{l}8,6 \\
8,7\end{array}$ & $\begin{array}{l}0,00 * * * \\
0,00 * * *\end{array}$ \\
\hline
\end{tabular}

coping: análise lógica $(F=3,65 ; p=0,01)$, busca de guia $(F$ $=8,57 ; p=0,00)$ e descarga emocional $(F=8,70 ; p=0,00)$. Essas diferenças mostraram-se significativas quando foram comparadas escolas públicas, particulares e conveniadas, demonstrando que os adolescentes das escolas particulares (terceiro ano e cursinho), em comparação a seus colegas que frequentavam outros tipos de escola (conveniadas ou públicas), foram os que mais utilizaram estratégias de enfrentamento do tipo análise lógica $(F=3,65 ; p=0,01)$, busca de guia $(F=8,57$; $p=0,00)$ e descarga emocional $(F=8,70 ; p=0,00)$ como forma de enfrentar o evento estressante vestibular.

Verificou-se que houve diferença significativa ao analisar as psicopatologias em relação a sexo, visto que as meninas relataram mais psicopatologias do tipo internalizante $(F=$ $12,52 ; p=0,00)$ e os meninos, do tipo externalizante $(F=$ $4,74 ; p=0,03)$. Contudo, neste estudo não foram observadas diferenças estatisticamente significativas para o total de condutas psicopatológicas em relação a sexo. A análise da variável psicopatologia por faixa etária dos vestibulandos também não mostrou diferenças estatisticamente significativas.

Ao comparar as psicopatologias internalizantes, externalizantes e total em relação ao tipo de escola, observouse diferença significativa quanto ao total de condutas psicopatológicas apresentadas pelos jovens e o tipo de escola que frequentavam $(F=3,24 ; p=0,02)$. Os que estavam frequentando o terceiro ano do ensino médio das escolas particulares $(M$ $=58,57 ; D P=9,08)$ tenderam a apresentar mais condutas psicopatológicas que os provenientes de outros tipos de escola, ou seja, os que frequentavam o terceiro ano da escola pública $(M$ $=46,15 ; D P=6,43)$, o terceiro ano da escola conveniada $(M=$ $55,91 ; D P=7,43)$ e o cursinho particular $(M=54,10 ; D P=8,18)$.

\section{Análise de regressão linear entre uso de coping e psicopatologias}

Para analisar a relação existente entre as estratégias de coping e o desenvolvimento de psicopatologias, utilizou-se o cálculo de regressão linear (Tabela 3). Com isso, objetivou-se verificar se o desenvolvimento de psicopatologias internalizantes, externalizantes ou o total de condutas psicopatológicas foi influenciado pelo tipo de estratégia de coping utilizado pelos adolescentes para enfrentar o vestibular.

Percebe-se que o uso de análise lógica e a aceitação e resignação como forma de enfrentamento de problemas explica em $16 \%$ o autorrelato de problemas externalizantes; o uso de aceitação e resignação e descarga emocional está relacionado com o desenvolvimento de psicopatologias internalizantes em um nível de 14\%; em geral, as estratégias de coping reavaliação positiva, aceitação e resignação e descarga emocional explicam uma variância de $22 \%$ no autorrelato de condutas psicopatológicas.

Entretanto, ao analisar o uso do conjunto de estratégias de coping segundo a categoria, estratégias de aproximação e de evitação, influenciou o desenvolvimento geral de condutas psicopatológicas, verificou-se que o conjunto de estratégias evitativas explicou de forma direta o desenvolvimento de psicopatologia contribuindo com beta $=0,463$, enquanto o conjunto de estratégias de aproximação explicou de forma inversa a manifestação do total de condutas psicopatológicas com beta $=-0,209$. Em conjunto, as estratégias de aproximação e evitação do problema contribuem em $18 \%$ na explicação da variância do total em condutas psicopatológicas. Contudo, parece que enquanto as estratégias de evitação do problema constituem um fator de risco para o desenvolvimento de psicopatologia, as estratégias de aproximação do problema podem ser consideradas como um fator de proteção.

\section{Discussão}

O objetivo principal deste trabalho foi investigar o uso de estratégias de coping por adolescentes vestibulandos e sua 
possível repercussão na saúde mental desses indivíduos. Ao descrever pesquisa realizada com uma população adolescente espanhola, Zanini e Forns (2004) apontaram que as meninas utilizaram significativamente mais estratégias de enfrentamento para resolver seus problemas que os meninos. Esse dado foi corroborado neste estudo, que demonstrou maior uso de estratégias de coping por parte da população feminina quando comparada com seus colegas do sexo masculino. Esse uso aconteceu de forma significativa para as respostas de coping de aproximação análise lógica, reavaliação positiva e busca de guia.

Holahan et al. (1996) destacam o uso mais frequente pelas meninas das estratégias análise lógica e descarga emocional, o que, segundo Sans (2005), pode estar associado à maior maturidade psicológica que elas apresentam em comparação aos meninos. Em outro estudo, Câmara e Carlotto (2007) relataram mais uso de estratégias de resolução de problemas pelas meninas e uma associação do uso destas estratégias com bem estar psicológico. Por outro lado, Larrea (2005) e Marcelli e Braconnier (2007) demonstraram que as meninas sentem mais necessidade de buscar apoio social para o enfrentamento de problemas, argumentação confirmada nesta pesquisa, pois a busca de guia foi significativamente maior em meninas.

A descarga emocional também foi encontrada como estratégia mais utilizada pelas meninas, o que pode ser um fator de risco para esta população. Embora o tipo de enfrentamento representado por comportamentos do tipo descarga emocional possa ser característico da adolescência e, em especial, da população feminina, o uso significativamente maior deste tipo de estratégia como forma de enfrentamento de problemas pode ser representativo de risco para este grupo, uma vez que, de acordo com Compas (1987) e Dell'Aglio e Hutz (2002), o uso de estratégias evitativas pode ser indicativo de risco psicológico.

Em seu estudo, ao relacionar a idade com o uso de estratégias de coping, Zanini e Forns (2004) constataram a existência de diferenças significativas tanto na qualidade como na quantidade de estratégias de coping utilizadas por adolescentes de diferentes idades. Em contrapartida, na presente pesquisa não foram encontradas diferenças no uso de coping entre adolescentes com idades variando entre 16 e 19 anos. Existem duas hipóteses que podem explicar esses resultados. Uma está relacionada ao número reduzido de indivíduos na amostra e a outra, ao fato de que algumas teorias do desenvolvimento não prevêem diferenças entre idades tão próximas quanto a da população estudada para o uso das estratégias de coping. Neste estudo, foram encontradas diferenças significativas por idade apenas para a percepção do problema e não para o uso de coping.

Verificou-se que existiram diferenças significativas entre os jovens dos diferentes tipos de escola examinados ao lidar com o evento estressante vestibular. Os jovens que estudavam em escolas da rede particular, tanto os que cursavam o terceiro ano do ensino médio quanto os alunos do cursinho preparatório para o vestibular, usaram significativamente mais estratégias evitativas, como busca de guia e descarga emocional. Isso pode indicar que esses jovens esforçam-se mais para enfrentar esse problema específico (vestibular). A provável explicação para esse resultado talvez seja a grande importância que as escolas particulares atribuem ao ingresso do aluno em determinadas universidades, tidas como centros de excelência, e a coação que sofrem estes estudantes em função da seleção do vestibular, principalmente aqueles que já tentaram antes e ainda não conseguiram ingressar no curso superior. Além disso, a política de algumas escolas e a percepção social de que sua qualidade de ensino estão voltadas para o número de alunos aprovados no vestibular para cursos e universidades consideradas de renome. Além da coação exercida por determinados tipos de instituições de ensino, fato constatado pela exposição de listas de aprovados, as famílias são coniventes com esta situação e, às vezes, sua cobrança pode ser ainda maior (Silvestre, 2004). Nesse sentido, não se pode ignorar a escolha realizada por pais e jovens no sentido de ingressar em escolas com esse perfil de política educacional representante de um contexto social específico e valores determinados.

Relacionando o sexo e o desenvolvimento de psicopatologias, constatou-se que as meninas desenvolveram mais síndromes internalizantes e os meninos, mais síndromes externalizantes, dados que corroboram aqueles descritos por Achenbach (1991; 1995) em ambas as síndromes, assim como os de Sans (2005) sobre a maior prevalência de depressão em mulheres e cujo início reporta à adolescência. Também confirmam o estudo de Soares e Martins (2010), que relataram índices mais elevados de ansiedade-traço e estado em mulheres submetidas ao exame do vestibular quando comparadas com os homens.

Nesta pesquisa foi demonstrado que as estratégias de coping aceitação e resignação e descarga emocional predispõem ao aparecimento de problemas de conduta internalizantes, enquanto as estratégias de coping análise lógica (aproximação) e aceitação e resignação (evitação) predispõem ao autorrelato de problemas externalizantes. Tais dados corroboram estudos anteriores que descrevem o fator desadaptativo do uso de estratégias de evitação do problema e sua relação com problemas psicopatológicos (Zanini \& Forns, 2004). Além disso, estudos transculturais também demonstraram associação entre estratégias de análise lógica e psicopatologias nas culturas espanhola (Zanini \& Forns, 2004) e brasileira (Borges, 2007), o que demonstra a necessidade de uma avaliação transcultural das estratégias de coping e sua relação com a saúde mental dos indivíduos.

Por outro lado, quando da análise em conjunto, observa-se que também para os adolescentes vestibulandos estudados na presente pesquisa, o uso de coping evitativo está relacionado com problemas de conduta e psicopatologias, enquanto o uso de coping ativo ou de aproximação mostrou-se um fator de proteção para a saúde mental dos indivíduos. Este dado está em concordância com o debatido na literatura nacional sobre coping (Antoniazzi et al., 1998; Clarke, 2006) e evidencia a importância do desenvolvimento de programas de intervenção ou prevenção baseados no desenvolvimento de habilidades de coping.

\section{Considerações finais}

A principal contribuição deste trabalho refere-se ao estudo da relação entre as estratégias de enfrentamento de problemas e a manifestação de comportamentos desadaptados relacionados ao enfrentamento do vestibular. Os resultados deste estudo põem em evidência a relação entre vestibular como evento estressante e saúde mental dos adolescentes. Os resultados mostram o 
risco psicopatológico vivenciado por adolescentes de escolas particulares que relatam interesse em realizar o vestibular, tendo em vista o maior índice de mal-estar psicológico relatados por este grupo de adolescentes em comparação com os outros (de escolas conveniadas e públicas). Nesse sentido, ressalta-se a necessidade de mudança na concepção de qualidade de ensino que tenha como único critério a aprovação no vestibular e não o bom desenvolvimento e a adaptação biopsicossocial do adolescente, em associação a uma mudança de política de acesso ao ensino superior. Em adição a esta informação, ressalta-se o fato de que o uso de evitação como forma de enfrentamento de problemas também se apresenta como fator de risco para a saúde mental, enquanto o enfrentamento direto dos problemas poderia constituir-se como um fator de proteção. Dentro de uma perspectiva educacional que abarcasse o desenvolvimento global do adolescente, a possibilidade de trabalho com as formas como esses indivíduos vivenciam e enfrentam seus problemas também poderiam ser de grande relevância para seu bom desenvolvimento.

Por fim, cabe ressaltar a necessidade de novas pesquisas que avaliem os aspectos trabalhados neste estudo, estendendo-os a outras populações e contextos socioculturais que não os das escolas e da cidade de Goiânia, em que o presente estudo foi desenvolvido, envolvendo maior número de participantes a fim de verificar a generalização destes dados.

\section{Referências}

Achenbach, T. M. (1991). Manual for the youth self-report and 1991 profile. Burlington: University of Vermont.

Achenbach, T. M. (1995). Developmental issues in assessment, taxonomy and diagnosis of child and adolescent psychopathology. In D. Cichetti \& D. J. Cohen (Orgs.), Developmental psychopathology (Vol. 1, pp. 57-80). New York: Wiley and Sons.

American Psychiatric Association (2002). Manual diagnóstico e estatístico de transtornos mentais - DSM-IV-TR (4를 ed., C. Dornelles, Trad.). Porto Alegre: Artmed.

Antoniazzi, A. S., Dell Aglio, D. D., \& Bandeira, D. R. (1998). A evolução do conceito de coping: uma revisão teórica. Estudos de Psicologia, 3(2), 273-294. Recuperado de http://www.scielo.br/scielo.php?script=sci_ arttext\&pid=S1413-94X1998000200006\&lng=en\&nrm=iso\&tlng=pt

Bordin, I. A. S., Mari, J. J., \& Carero, M. F. (1995) Validação da versão brasileira do Child Behavior Checklist (CBCL) Inventário de comportamentos da infầncia e adolescência: dados preliminares. Revista ABP-APAL, 17(2), 55-66.

Borges, J. M. (2007). Coping e saúde mental de grávidas adolescentes participantes do Projeto Meninas de Luz (Dissertação de mestrado não publicada). Pontifícia Universidade Católica de Goiás, Goiânia.

Calais, S. L., Andrade, L. M. B., \& Lipp, M. E. N. (2003). Diferenças de sexo e escolaridade na manifestação do stress em adultos jovens. Psicologia: Reflexão \& Crítica, 16, 257-263.

Câmara, S. G., \& Carlotto, M. S. (2007). Coping e gênero em adolescentes. Psicologia em Estudo, 12(1), 87-93.

Clarke, A. T. (2006). Coping with interpersonal stress and psychosocial health among children and adolescents: a meta-analysis. Journal of Youth and Adolescence, 35(1), 10-23.

Compas, B. E. (1987). Coping with stress during chilhood and adolescence. Psychological Bulletin, 101(3), 393-403.

Dell'Aglio, D. D. (2003). O processo de coping em crianças e adolescentes: adaptação e desenvolvimento. Temas em Psicologia, 11(1), 38-45.
Recuperado de http://www.sbponline.org.br/revista2/vol11n1/art04_t.htm Dell'Aglio, D. D., \& Hutz, C. S. (2002). Estratégias de coping de crianças e adolescentes em eventos estressantes com pares e com adultos. Psicologia USP, 13(2), 203-225. Recuperado de http://www.scielo.br/scielo. php?script=sci_arttext\&pid=S0103-65642002000200012\&lng=es\&nrm= iso\&tlng=es

Faria, J. B. de, \& Seidl, E. M. F. (2005). Religiosidade e enfrentamento em contextos de saúde e doença: revisão da literatura. Psicologia: Reflexão e Crítica, 18(3), 381-389.

Holahan, C. J., Moos, R. H., \& Schaefer, J. A. (1996). Coping, stress resistance and growth: conceptualization adaptive functioning. In M. Zeidner \& N. S. Endler (Orgs.), Handbook of coping: theory, research, applications (pp. 24-43). New York: John Wiley \& Sons.

Larrea, S. (2005). Las relaciones entre padres e hijos adolescentes. In E. Domènech-Llaberia (Org.), Actualizaciones en psicología y psicopatología de la adolescencia (pp. 185-200). Bellaterra: Universitat Autónoma de Barcelona.

Lazarus, R. S., \& Folkman, S. (1984). Stress, appraisal and coping. New York: Springer.

Lei $n^{\circ} 5.540$, de 28 de novembro de 1968. (1968, 23 novembro). Fixa normas de organização e funcionamento do ensino superior e sua articulação com a escola média, e dá outras providências. Recuperado de http://www.planalto. gov.br/Ccivil_03/LEIS/L5540.htm

Lei $n^{\circ}$ 9.394, de 20 de dezembro de 1996. (1996, 20 dezembro). Estabelece as diretrizes e bases da educação nacional. Recuperado de http://www.planalto. gov.br/ccivil_03/LEIS/L9394.htm

Marcelli, D., \& Braconnier, A. (2007). Adolescência e psicopatologia (6 $6^{\mathrm{a}}$ ed., F. Murad, Trad.). Porto Alegre: Artmed.

Moos, R. H. (1993). Coping response inventory youth form - professional manual. Odessa: PAR Psychological Assessment Resources.

Peluso, M. A. M., Savalli, C., Cúri, M., Gorenstein, C., \& Andrade, L. H. (2010). Alterações de humor ao longo da preparação para vestibular - um estudo longitudinal. Revista Brasileira de Psiquiatria, 32(1), 30-36.

Pesce, R. P., Assis, S. G., Santos, N., \& Oliveira, R. V. C. (2004). Risco e proteção: em busca de um equilíbrio promotor de resiliência. Psicologia: Teoria e Pesquisa, 20(2), 135-143. Recuperado de http://www.bvsde.paho. org/bvsacd/cd26/a06v20n2.pdf

Reitz, E., Dekovic, M., \& Meijer, A. M. (2005). The structure and stability of externalizing and internalizing problem behavior during early adolescence. Journal of Youth and Adolescence, 34(6), 577-588.

Rocha, C. R. S., Rossini, S., \& Reimão, R. (2010). Sleep disorders in high school and pre-university students. Arquivos de Neuro-Psiquiatria, 68(6), 903-907.

Sans, J. C. (2005). El impacto de la pubertad sobre el desarrollo del adolescente. In E. Domènech-Llaberia (Org.), Actualizaciones em psicología y psicopatología de la adolescencia (pp. 39-52). Bellaterra: Universitat Autónoma de Barcelona.

Savoia, M. G. (1999). Escala de eventos vitais e de estratégias de enfrentamento (coping). Revista de Psiquiatria Clínica, 26(2), 56-67. Recuperado de http:// www.hcnet.usp.br/ipq/revista/r262/artigo(57).htm

Silva, M. D. F. D. T., Aznar-Farias, M., Silvares, E. F. M., \& Arantes, M. C. (2008). Adversidade familiar e problemas comportamentais entre adolescentes infratores e não-infratores. Psicologia em Estudo, 13(4), 791-798.

Silvestre, G. (2004). Vestibular sem sofrimento: o segredo dos vencedores. Belo Horizonte: Gutenberg.

Soares, A. B., \& Martins, J. S. R. (2010). Ansiedade dos estudantes diante da expectativa do exame vestibular. Paidéia, 20(45), 57-62.

Sparta, M., \& Gomes, W. B. (2005). Importância atribuída ao ingresso na educação superior por alunos do ensino médio. Revista Brasileira de Orientação Profissional, 6(2), 45-53.

Stevens, G. W. J. M., Vollebergh, W. A. M., Pels, T. V. M., \& Crijnen, A. A. M. (2005). Predicting internalizing problems in Moroccan immigrant adolescents 
154 L. S. D. Silva \& D. S. Zanini

in the Netherlands. Social Psychiatry and Psychiatry Epidemiology, 40(12), 1003-1011.

Wagner, A., Falcke, D., Silveira, L. M. B. O., \& Mosmann, C. P. (2002). A comunicação em famílias com filhos adolescentes. Psicologia em Estudo, 7(1), 75-80. Recuperado de http://www.scielo.br:/scielo.php?script=scielo arttext\&pid=51413-7372200200100010

Zanini, D. S., \& Forns, M. (2004). Coping y psicopatología: comparación entre adolescentes de la muestra general y sub-clínica. Psiquiatria.com, $8(2)$. Recuperado de http://hdl.handle.net/10401/774

Zanini, D. S., Forns, M., \& Kirchner, T (2005). Coping response and problem appraisal in Spanish adolescents. Perceptual and Motor Skills, 100, 153-166.

Zanini, D. S., Mendonça, H., Forns, M., \& Kirchner, T. (2010). Psychometric properties of the coping response inventory with brazilian adolescents. Psychological Reports, 107(2), 617-628.

Lorena Soares Dias e Silva, Mestre em Psicologia pela Pontifícia Universidade Católica de Goiás, é psicóloga escolar no Colégio Expovest Júnior e no Colégio Expocursos, Expovest JR e Expocursos. Endereço para correspondência: Av. Berlim, no 1100, Lt 1-A, cs 15, Rs. Gramado. Jardim Europa. Goiânia-GO. CEP:74330010. Tels.: (62)3932-0819/(62)9311-8696. E-mail: lorenapsicol@yahoo.com.br

Daniela S. Zanini, Doutora em Psicologia Clínica e da Saúde pela Universidad de Barcelona, pós-doutora em Psicologia Clínica e da Saúde pela Universidade de Barcelona, é professora na Pontifícia Universidade Católica de Goiás. E-mail: dazanini@yahoo.com 